\title{
Routine Molecular Screening of Patients with Advanced Non-SmallCell Lung Cancer in Circulating Cell-Free DNA at Diagnosis and During Progression Using OncoBEAM ${ }^{\mathrm{TM}}$ EGFR V2 and NGS Technologies
}

\author{
Jessica Garcia ${ }^{1,2}$ - Arnaud Gauthier ${ }^{1} \cdot$ Gaëlle Lescuyer ${ }^{1,2}$ - David Barthelemy ${ }^{1,2}$. Florence Geiguer ${ }^{1,2}$. \\ Julie Balandier ${ }^{1,2} \cdot$ Daniel L. Edelstein $^{3} \cdot$ Frederick S. Jones $^{3} \cdot$ Frank Holtrup $^{3} \cdot$ Mickael Duruisseau $^{4}$. \\ Emmanuel Grolleau $^{6} \cdot$ Claire Rodriguez-Lafrasse $^{1} \cdot$ Patrick Merle $^{5} \cdot$ Sébastien Couraud $^{2,6,7} \cdot$ Léa Payen $^{1,2,8} \mathbb{D}$
}

Accepted: 3 February 2021 / Published online: 3 March 2021

(c) The Author(s) 2021

\begin{abstract}
Background and Objectives The use of ultra-sensitive diagnostic tests to detect clinically actionable somatic alterations within the gene encoding the epidermal growth factor receptor $(E G F R)$ within circulating cell-free DNA is an important first step in determining the eligibility of patients with non-small cell lung cancer to receive tyrosine kinase inhibitors.

Methods We present the clinical validation (accuracy, sensitivity, and specificity) of a highly sensitive OncoBEAM ${ }^{\mathrm{TM}} E G F R$ V2 test, which we compare to a custom next-generation sequencing assay, for the treatment of patients with non-small cell lung cancer with $E G F R$ tyrosine kinase inhibitor therapies. The OncoBEAM ${ }^{\mathrm{TM}}$ digital-polymerase chain reaction method detects 36 different $E G F R$ alterations in circulating cell-free DNA, whereas the next-generation sequencing assay covers major solid tumor oncodrivers. Of the 540 samples analyzed with the OncoBEAM ${ }^{\mathrm{TM}}$ EGFR V2 test, $42.4 \%$ of patients had undergone molecular testing at diagnosis $(N=229 / 540)$ and $57.7 \%$ of patients during disease progression $(N=311 / 540)$.

Results The sensitivity and specificity were measured for this BEAMing assay. The number of mutant beads and mutant allelic fraction were measured for each EGFR alteration and the level of detection was established at $0.1 \%$ for a median of 2861 genome equivalent (GE) in each reaction using HD780 horizon control DNA, as well as by an internal quality reference standard. Approximately 10\%, 27\%, and 63\% of the 540 samples contained $<1500 \mathrm{GE}$, a range of 1500-3000 GE, and $>3000 \mathrm{GE}$, which corresponded to a maximal assay sensitivity of $2.0 \%, 0.5-0.1 \%$, and $0.1-0.05 \%$ mutant allelic fraction, respectively. In a routine hospital setting, $11.4 \%$ of non-small cell lung cancer tumors were positive at diagnosis for EGFR alterations, while $43.7 \%$ samples harbored EGFR mutations at progression, among which $40.3 \%$ expressed EGFR resistance mutations after first-line tyrosine kinase inhibitor treatment with first- and second-generation drugs.

Conclusions The OncoBEAM ${ }^{\mathrm{TM}} E G F R$ V2 is a sensitive, robust, and accurate assay that delivers reproducible results. Nextgeneration sequencing and BEAMing technologies act complementarily in the routine molecular screening. We show that using a next-generation sequencing assay, despite its lower sensitivity, enables the identification of rare EGFR alterations or resistance mechanisms (mutation, deletion, insertion, and copy number variation) to orient first- and second-line treatments.
\end{abstract}

\section{Introduction}

Lung cancer is the leading cause of cancer-related deaths worldwide with a 5 -year survival rate of $15 \%$. Non-small cell lung cancer (NSCLC) is the major subtype, encompassing $85 \%$ of all lung cancers [1]. Significant advances have been made to identify tumor biomarkers for the development of highly effective therapies that target specific tumor somatic alterations, revolutionizing NSCLC management [2]. These

\section{Léa Payen}

Lea.payen-gay@chu-lyon.fr

Extended author information available on the last page of the article include mutations/deletions/rearrangements in genes encoding the epidermal growth factor receptor (EGFR), the echinoderm microtubule-associated protein-like 4-anaplastic lymphoma kinase (EMLA-ALK), and the hepatocyte growth factor ( $H G F$ or $M E T)$. Tyrosine kinase inhibitor (TKI) therapies, which prevent the growth of tumors driven by signaling pathways involving these proteins, have greatly improved the survival of patients with NSCLC [3-6].

At initial diagnosis, somatic EGFR alterations have been observed in $10-15 \%$ and $40-55 \%$ of NSCLC cases in Caucasian and Asian populations, respectively [7]. Such changes lead to constitutive tyrosine kinase activation, promoting growth and metastatic spread of cancer cells. EGFR 


\section{Key Points}

Detection of somatic alterations within the gene encoding the epidermal growth factor receptor (EGFR) is an important first step to determining the eligibility of patients with non-small cell lung cancer to receiving targeted therapies.

The development of ultra-sensitive diagnostic tests that detect EGFR mutations in circulating cell-free DNA can improve the identification of candidates eligible for tyrosine kinase inhibitors at diagnosis in the early stages of non-small cell lung cancer.

The OncoBEAM ${ }^{\mathrm{TM}}$ EGFR V2 is a sensitive, robust, and accurate assay that delivers reproducible results. Nextgeneration sequencing and BEAMing technologies act complementarily in the routine molecular screening.

alterations are predictive of response to EGFR-TKIs, including the reversible first generation of TKIs (gefitinib and erlotinib), the irreversible second generation of TKIs (afatinib and dacomitinib), and the irreversible third generation of TKIs (osimertinib). The two most prevalent sensitizing EGFR alterations (constituting over $80 \%$ of all mutations) are small in-frame deletions within exon 19 (delEX19) and a single-point mutation in exon 21 , namely p.L858R, both residing within the tyrosine kinase domain of $E G F R[8,8]$. Before the first-line use of third-generation EGFR TKIs, such as osimertinib, patients with NSCLC generally develop resistance to first- and second-generation TKIs within 9-12 months of treatment. This resistance is caused by (1) the emergence of clones having the secondary resistance mutation EGFR p.T790M (50\% of cases) [10, 11], (2) the activation of parallel signaling pathways via amplifications, mutations, or fusions involving the $M E T, E R B B 2$, and $A L K$ genes, (3) the phenotypic transformation from NSCLC to small cell lung cancer ( $15 \%$ of cases), and (4) the epithelialto-mesenchymal transition ( $2 \%$ of cases) [12-14].

Molecular diagnostic testing for somatic alterations is recommended at diagnosis for advanced NSCLC, and is usually performed on archival formalin-fixed paraffin-embedded tumor tissues. However, in clinical practice, repeating such tissue biopsies during disease progression is often a challenge, even highlighted in large clinical trials [15]. Moreover, this invasive sampling procedure is not appropriate for patients during disease progression as it can cause undue health complications, particularly in patients with comorbidities, and may further delay treatment administration. Adverse event rates can reach $17.1 \%$ for thoracic biopsies [15]. Considering these limitations, less invasive and more convenient techniques to monitor EGFR-TKI treatments in NSCLC are urgently needed.

Over the last decade, significant technological advances have enabled the detection of tumor biomarkers that assist treatment-management decisions using materials present in blood samples of patients with cancer. Tumors release fragments of circulating cell-free DNA (cfDNA) into the bloodstream, and the blood sample is collectively known as "liquid biopsy" [16, 17]. Non-invasive cfDNA genotyping is a cost-effective alternative to tissue biopsy, especially when surgical procedures cannot yield tissue samples of sufficient quantity and/or quality for testing. The clinical potential of non-invasive material, such as cfDNA, has been demonstrated in many studies including those examining therapeutic response, monitoring, and residual disease detection [18]. Nevertheless, relapse is inevitable in patients treated with TKIs, and biopsies are necessary to identify the emergent tumoral clones underlying resistance to treatment via the selection or acquisition of additional somatic alterations. However, it can be challenging at diagnosis and during progression to obtain sufficient quantities of tumor biopsy material to generate accurate and reproducible results by molecular testing. When re-biopsy is not feasible, the GFCO "Groupe Francophone de Cytogénomique Oncologique" recommends molecular testing of cfDNA in France [19-21].

Among all assays dedicated to detecting genetic alterations in cfDNA, we focused on the clinical performance of the OncoBEAM ${ }^{\mathrm{TM}} E G F R$ V2 digital PCR kit. The assay was expanded to cover 36 prevalent $E G F R$ mutations found in NSCLC, in comparison to the original OncoBEAM ${ }^{\mathrm{TM}}$ $E G F R \mathrm{~V} 1$ assay used in the AURA trial [22]. The assay relying on cfDNA analysis was optimized to identify EGFRpositive tumors to improve diagnosis at early stages, as well as to monitor treatment efficacy, and to determine resistance mechanisms associated with the selection of EGFR p.T790M or EGFR p.C797S mutations during progression. Clinical performance was assessed by comparing the BEAMing assay with the next-generation sequencing (NGS) assay, in a retrospective study of 659 cfDNA samples collected during routine management of patients with NSCLC at the Hospices Civils of Lyon (France). This work is the first study to clinically validate the OncoBEAM ${ }^{\mathrm{TM}} E G F R$ $\mathrm{V} 2$ assay workflow using internal and external quality controls to determine the accuracy, sensitivity, and specificity of this assay.

\section{Materials and Methods}

\subsection{Ethical Considerations and Patient Inclusion}

All internal and external (from rural hospitals) samples collected/sent to the laboratory for EGFR routine screening 
between September 2018 and February 2020 were included in this retrospective cohort study. For each sample, medical data were collected through a mandatory prescription sheet attached to each sample and edited by the prescribing physician.

As recommended, blood screening was performed either at diagnosis or during progression alongside a regular follow-up computed tomography scan (usually performed quarterly). At diagnosis, all tumors were histologically characterized on formalin-fixed paraffin-embedded specimens and an $E G F R$-sensitizing mutation detection was performed either on formalin-fixed paraffin-embedded tumor samples or by using cfDNA in the case of tumor tissue genotyping failure as part of the routine practice. In this cohort, a subset of patients (with a confirmatory EGFR tumor mutation-positive result at diagnosis) underwent several serial cfDNA analyses as recommended by their physician because of a suspicion of disease progression at the time of the computed tomography scan. During progression, EGFR mutation-positive patients were treated by TKI treatment in the first intention, and molecular resistance profile was researched.

\section{2 cfDNA Collection}

Circulating cell-free DNA was extracted from $4 \mathrm{~mL}$ or $8 \mathrm{~mL}$ of plasma using the QIAamp Circulating Nucleic Acid Kit (Cat No 55114; Qiagen, Valencia, CA, USA), with a Qiagen vacuum manifold following the manufacturer's instructions [11]. Circulating cell-free DNA samples were quantified using the Qubit dsDNA HS assay (Q32854; Life Technologies, Carlsbad, CA, USA) and then stored at $-80^{\circ} \mathrm{C}$ until further use.

\subsection{Detection EGFR of Alterations Using the BEAMing Assay}

Circulating cell-free DNA was analyzed for $E G F R$ alterations using the OncoBEAM ${ }^{\mathrm{TM}} E G F R$ V2 kit (Cat No ZR150220; Sysmex Inostics, Hamburg, Germany) according to the manufacturer's instructions. The OncoBEAM ${ }^{\mathrm{TM}}$ $E G F R$ V2 kit is a quantitative assay that detects 36 alterations in the human EGFR gene in exons 18, 19, 20, and 21. These include p.G719X, p.L858R, and p.L861Q mutations, 27 of the most prevalent deletions in exon 19, and p.T790M and p.C797S EGFR resistance mutations (Table 1 of the Electronic Supplementary Material [ESM]). In our study, the range of DNA quantities used as input for the OncoBEAM EGFR V2 assay was between 2.88 and $800.00 \mathrm{ng}$ of DNA. The threshold of positivity, specific to detected alterations, was defined using three parameters: (1) the mutant allelic fraction (MAF) had to exceed $0.02 \%$; (2) the absolute number of mutant beads had to be greater than 50; and (3) the shape of the bead distribution had to be clustered in the flow cytometry plot. Two internal controls were included in each experiment. A sample adequacy control (SAC) was included in each experiment to accurately quantify cfDNA input in each sample and served as an independent patient assay using the BEAMing technology workflow. A known amount of synthetic SAC DNA, present in the multiplex polymerase chain reaction (PCR), was used to draw a correlation between BEAMing results and endogenous HBB ( $\beta$-hemoglobin) BEAMing results of each sample. The sequence of the SAC was derived from the human endogenous HBB with several modifications. HBB and SAC were amplified using the same primers as a separate amplicon for each sample and hybridized with a probe mix separating them into two populations. From the ratio of the two populations, the DNA input in the multiplex PCR was automatically calculated as copies/sample by the OncoBEAM kit software and expressed in genome equivalent (GE), corresponding to $3.3 \mathrm{pg}$ of cfDNA. Detected mutations were determined as the MAF percentage in the context of cfDNA input expressed in GE.

\subsection{Detection of EGFR Alterations Using NGS}

For the NGS assay, libraries were prepared from 10 to 50 ng of cfDNA, using a custom capture-based technology provided by Sophia Genetics (Geneva, Switzerland). In total, an oncology panel of 66 genes (principal exons for all oncodriver genes, including TP53, EGFR, BRAF, NRAS, KRAS, $P I K 3 C A$, and MET, as well as others) was covered by 2400 probes $(75 \mathrm{~kb})$ [see ESM].

\subsection{Statistical Analysis}

Descriptive statistics were performed using the GraphPad InStat software, version 8.4.2 (La Jolla, CA, USA).

\section{Results}

\subsection{Correlation Between cfDNA Levels Measured with Qubit and Calculated with SAC Methodology}

The OncoBEAM ${ }^{\mathrm{TM}}$ EGFR V2 assay workflow included the quantification of cfDNA in each sample as an independent PCR reaction, and the use of SAC to convert mutant allelic fractions (MAF, \%) into a known amount of mutant molecules, to achieve more stringent quality control in cfDNA samples from patients. This assay leads to the ultrasensitive detection of EGFR alterations ( $0.02 \% \mathrm{MAF}$ ) owing to multiple parallel PCR reactions and the use of oil droplets to create millions of individual compartments for digital amplification. The GE content reflects the cfDNA quantity input 
Table 1 Sample description of the cohort studied for the detection of EGFR alterations in cfDNA of patients with NSCLC plasma samples using the OncoBEAM ${ }^{\mathrm{TM}}$ EGFR $\mathrm{V} 2$ and/or NGS assays

\begin{tabular}{|c|c|c|c|c|c|c|}
\hline & Diagnosis & Missing & Progression & $N$ & $\%$ at diagnosis & $\begin{array}{l}\% \text { during } \\
\text { progression }\end{array}$ \\
\hline \multicolumn{7}{|l|}{ General information } \\
\hline Women $(N)$ & 132 & 12 & 236 & 380 & 20.0 & 35.8 \\
\hline $\operatorname{Men}(N)$ & 117 & 22 & 140 & 279 & 17.8 & 21.2 \\
\hline Internal recruitment & 168 & 16 & 175 & 359 & 25.5 & 26.6 \\
\hline External recruitment & 81 & 18 & 201 & 300 & 12.3 & 30.5 \\
\hline \multicolumn{7}{|l|}{ Histological type } \\
\hline Adenocarcinoma & 120 & 12 & 308 & 440 & 18.2 & 46.7 \\
\hline Missing & 113 & 21 & 54 & 188 & 17.1 & 8.2 \\
\hline Other types & 16 & 1 & 14 & 31 & 2.4 & 2.1 \\
\hline \multicolumn{7}{|l|}{ Stage } \\
\hline Stage I & 0 & 0 & 1 & 1 & 0.0 & 0.0 \\
\hline Stage III & 5 & 0 & 4 & 9 & 0.8 & 0.6 \\
\hline Stage IV & 78 & 9 & 137 & 224 & 11.8 & 20.8 \\
\hline Missing & 166 & 25 & 234 & 425 & 25.2 & 35.5 \\
\hline
\end{tabular}

Data indicate whether samples were collected at the time of initial diagnosis, during progression, or not available $c f D N A$ circulating cell-free DNA, EGFR epidermal growth factor receptor, $N G S$ next-generation sequencing, $N S C L C$ non-small cell lung cancer

into the assay for each sample. The correlation between the GE content (from SAC reaction calculation) and the initial cfDNA level determined using the Qubit HS assay was evaluated based on the cfDNA samples (Fig. 1a). Depending on the quantity of cfDNA, the level in the first multiplex PCR was recalculated in a final volume of $125 \mu \mathrm{L}$ and compared with cfDNA input calculated in nanograms using the SAC. Figure 1a highlights a correlation between GE determination and Qubit level $\left(r^{2}=0.79\right.$, Pearson's test), whereas Fig. 1b shows a similar distribution of samples at diagnosis and during progression according to cfDNA input. The specificity and sensitivity of each range of cfDNA input is indicated in Table 2 and Fig. 1 of the ESM.

\section{2 cfDNA Molecular Testing of Clinical Samples from NSCLC Using the OncoBEAM ${ }^{\mathrm{TM}}$ EGFR V2 Assay}

Overall, we included 540/659 samples of cfDNA (82.0\%) in the OncoBEAM ${ }^{\mathrm{TM}} E G F R$ V2 assay, based on prescription indications. We excluded 119 samples that lacked information on sampling time (at diagnosis or progression) or did not require EGFR BEAMing testing owing to KRAS, BRAF mutations, $A L K$, or ROS1 fusions, previously found in the tissue biopsy. The recruitment of samples was well balanced between internal $(N=359)$ and external sampling $(N=300)$ (Table 1). The majority of patients included harbored an adenocarcinoma at an advanced stage of metastasis (stage IV).
Of the 540 samples analyzed, $42.4 \%$ of patients had undergone molecular testing at diagnosis $(N=229 / 540)$ and $57.5 \%$ of patients during disease progression under TKI treatment $(N=311 / 540)$ (Table 2). Among patients with progressive disease, $43.7 \%$ of samples showed $E G F R$ mutations and $40.3 \%$ had $E G F R$ resistance mutations after first-line TKI treatment with first- and second-generation EGFR-TKI drugs. The distribution of samples according to GE amounts of cfDNA is shown in Fig. 1b. Approximately, $10 \%, 27 \%$, and $63 \%$ of the samples contained less than 1500 GE, 1500-3000 GE, and more than $3000 \mathrm{GE}$, corresponding to a maximal sensitivity of $1.00 \%, 0.10 \%$, and $0.05 \%$ MAF, respectively (Fig. 1b). This is in agreement with the sensitivity of detection of the test according to cfDNA input. Genome equivalent content was determined by quality control (Table 3 of the ESM).

Overall, EGFR somatic alterations were found in $11.2 \%$ of cases at diagnosis $(N=26 / 229)$, primarily deletions in EGFR exon $19(4.7 \%, N=11 / 229)$ and EGFR p.L858R (3.9\%, $N=9 / 229)$ (Table 2A). The EGFR mutation showing the lowest prevalence was p.L861Q, which was detected in $1.70 \%$ of cases $(N=4 / 229)$; none of the patient samples had p.G719X mutations (0/229). Interestingly, the secondary EGFR p.T790M resistance point mutation was detected at diagnosis without $E G F R$ alteration in $0.9 \%$ of cases $(N=$ 2/229) (Table 2A).

During progression after first-line $E G F R$ TKI treatment, the deletions of exon 19 and p.L858R were detected at a higher frequency than at diagnosis $(22.2 \%$ and $14.5 \%$, respectively) (Table 2A). Finally, the EGFR p.T790M 


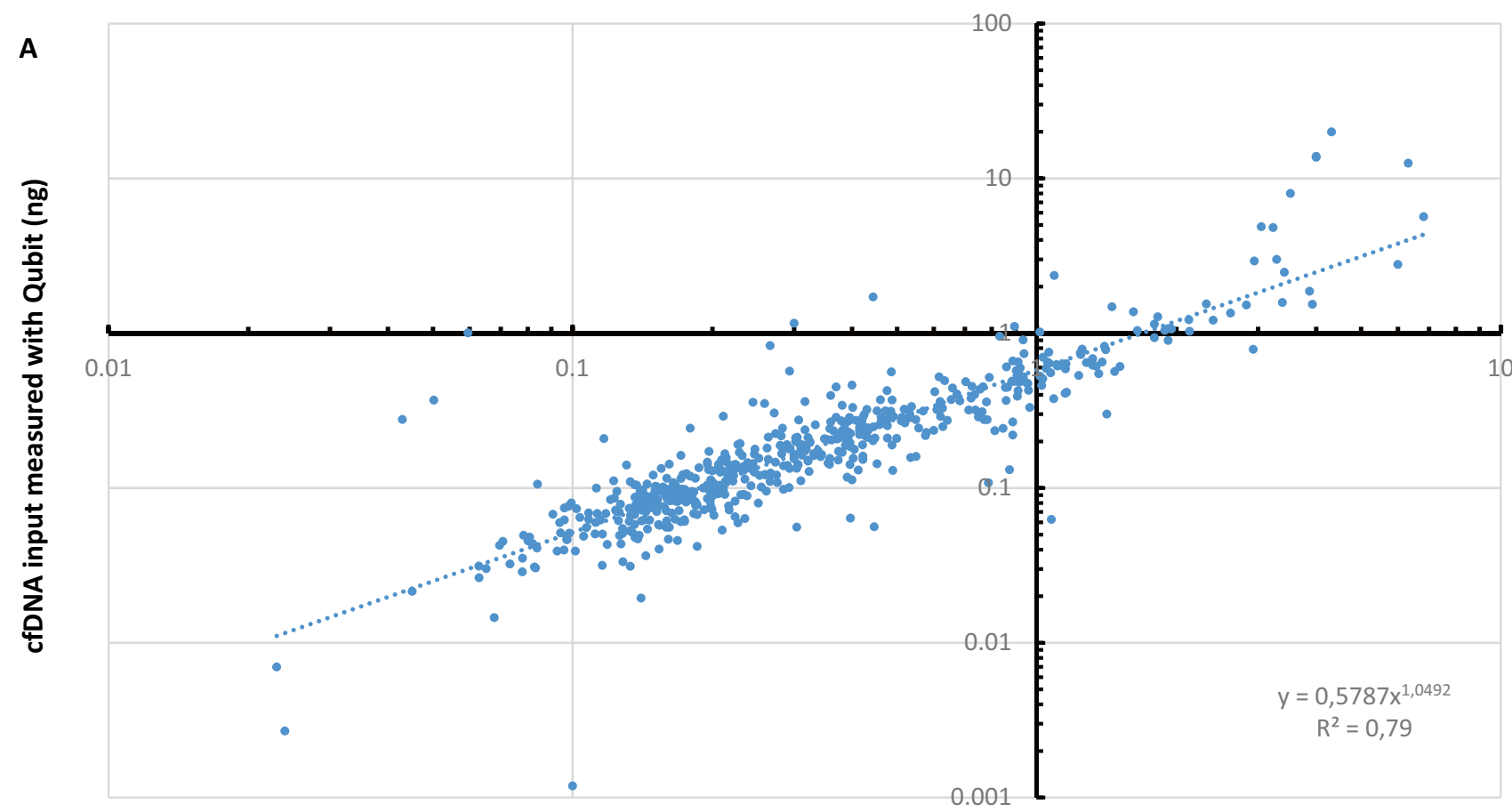

cfDNA input calculated with SAC result (ng)

B

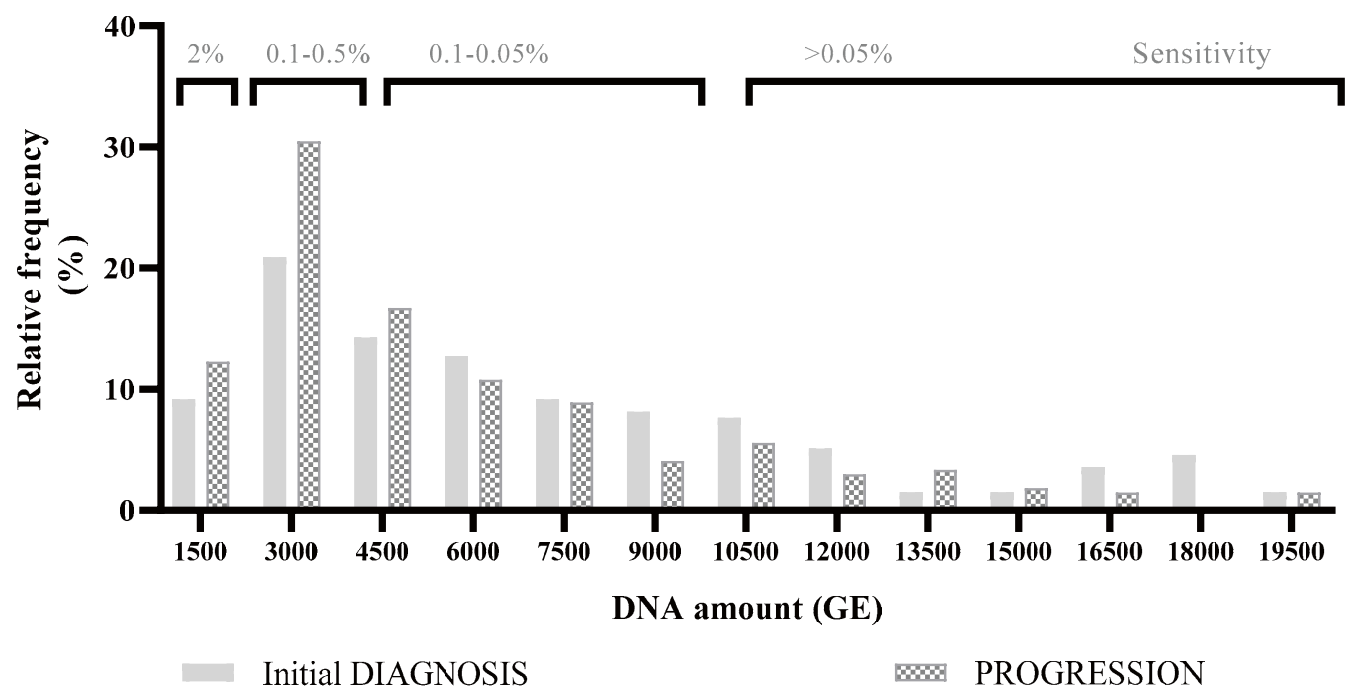

Fig. 1 Evaluation of the association between genome equivalent (GE) and input circulating cell-free DNA (cfDNA) level with clinical sample distribution according to GE. a Correlation between the input level of cfDNA (ng/ $\mu \mathrm{L})$ and the number of GEs. b Relative frequency

mutation was co-detected with its associated sensitizing EGFR mutation in $30.1 \%$ of cases (Table 2B). The secondary resistance mutation EGFR p.C797S was also detected in $4.1 \%(N=13 / 311)$ of cases (Table $2 \mathrm{~A})$, and generally in the absence of EGFR p.T790M (Table 2B). Using NGS assays, $60 \%$ of the EGFR alterations detected by the OncoBEAM ${ }^{\mathrm{TM}}$ $E G F R$ V2 assay were also detected. With the OncoBEAM ${ }^{\mathrm{TM}}$
(\%) of samples distributed according to the GE measured within the OncoBEAM EGFR V2 assay workflow for samples collected at diagnosis (gray) and at progression (black squares). EGFR epidermal growth factor receptor. SAC sample adequacy control

$E G F R$ V2 assay, $55 \%$ of MAF values of $E G F R$ alterations detected were below $1 \%$, whereas the NGS assay detected only $5 \%$ of cases with such low MAF values. Furthermore, for MAF values, the ranges were $1-5 \%$ and $5-10 \%$, the OncoBEAM $^{\mathrm{TM}}$ EGFR V2 assay detection level was $24 \%$ and $6 \%$, respectively, while NGS assay values were $52 \%$ and 
Table 2 Number of negative and positive cfDNA clinical samples for EGFR gene mutation screening that were analyzed with the OncoBEAM $^{\mathrm{TM}} E G F R$ V2 test at diagnosis or progression. Among the 660 samples, 540 samples were analyzed using the BEAMing assay

\begin{tabular}{|c|c|c|c|c|c|c|}
\hline \multicolumn{7}{|c|}{ A. General description per alteration } \\
\hline$E G F R$ alteration & $N$ (samples) & \multicolumn{2}{|c|}{ Wild-type $E G F R$ case } & \multicolumn{2}{|c|}{ At least one EGFR alteration } & $\begin{array}{l}\text { Positive (\%) with } \\
\text { one } E G F R \text { alteration }\end{array}$ \\
\hline \multicolumn{7}{|l|}{ Global } \\
\hline Diagnosis & 229 & \multicolumn{2}{|c|}{204} & \multicolumn{2}{|l|}{26} & 11.4 \\
\hline Progression & 311 & \multicolumn{2}{|c|}{175} & \multicolumn{2}{|l|}{$136^{\mathrm{c}}$} & 43.7 \\
\hline \multicolumn{7}{|l|}{ Diagnosis } \\
\hline$E G F R$ p.G719X (exon 18) & 229 & \multicolumn{2}{|c|}{229} & \multicolumn{2}{|l|}{0} & 0.0 \\
\hline Deletion of EGFR exon 19 & 229 & \multicolumn{2}{|c|}{218} & \multicolumn{2}{|l|}{11} & 4.8 \\
\hline$E G F R$ p.T790M (exon 20) & 229 & \multicolumn{2}{|c|}{227} & \multicolumn{2}{|l|}{$2^{\mathrm{b}}$} & 0.8 \\
\hline EGFR p.L858R (exon 21) & 229 & \multicolumn{2}{|c|}{220} & \multicolumn{2}{|l|}{9} & 3.9 \\
\hline EGFR p.L861Q (exon 21) & 229 & \multicolumn{2}{|c|}{225} & \multicolumn{2}{|l|}{4} & 1.7 \\
\hline EGFR p.C797S (exon20) & 229 & \multicolumn{2}{|c|}{229} & \multicolumn{2}{|l|}{0} & 0.0 \\
\hline \multicolumn{7}{|l|}{ Progression } \\
\hline$E G F R$ p.G719X (exon 18) & 311 & \multicolumn{2}{|c|}{306} & \multicolumn{2}{|l|}{5} & 1.6 \\
\hline Deletion of EGFR exon 19 & 311 & \multicolumn{2}{|c|}{242} & \multicolumn{2}{|l|}{69} & 22.2 \\
\hline$E G F R$ p.T790M (exon 20) & 311 & \multicolumn{2}{|c|}{265} & \multicolumn{2}{|l|}{$46^{\mathrm{a}}$} & 14.8 \\
\hline EGFR p.L858R (exon 21) & 311 & \multicolumn{2}{|c|}{266} & \multicolumn{2}{|l|}{45} & 14.5 \\
\hline EGFR p.L861Q (exon 21) & 311 & \multicolumn{2}{|c|}{304} & \multicolumn{2}{|l|}{7} & 2.3 \\
\hline EGFR p.C797S (exon 20) & 311 & \multicolumn{2}{|c|}{298} & \multicolumn{2}{|l|}{$13^{\mathrm{b}}$} & 4.2 \\
\hline B. Description of co-alterati & & & & & & \\
\hline & sensitizing & & $\begin{array}{l}\text { sensitizing } E G R F \\
+E G F R \text { p.T790M } \\
20)\end{array}$ & $\begin{array}{l}\text { ration } \\
\text { on }\end{array}$ & $\begin{array}{l}\text { EGFR alteration } \\
+E G F R \text { p.T790M } \\
(\text { exon } 20) \\
+E G F R \text { p.C797S } \\
(\text { exon } 20)\end{array}$ & $\begin{array}{l}E G F R \text { alteration } \\
+E G F R \text { p.C797S } \\
(\text { exon } 20)\end{array}$ \\
\hline Progression & & & & & & \\
\hline$N$ & 126 & & 38 & & 2 & 11 \\
\hline$\%$ & - & & 30.1 & & 1.5 & 8.7 \\
\hline
\end{tabular}

The number of samples with one or more EGFR alterations are indicated in the table; the frequency of positive samples with at least one EGFR alteration was tabulated as the given percentage

Samples (40.3\%) contained at least one EGFR-resistant alteration in the positive EGFR sensitizing samples

$c f D N A$ circulating cell-free DNA, EGFR epidermal growth factor receptor

${ }^{a}$ Eight without a sensitizing EGFR alteration

${ }^{\mathrm{b}}$ Two without a sensitizing EGFR alteration

${ }^{\mathrm{c}}$ At least one sensitizing or resistant EGFR alteration in the sample

7\%. In Fig. 2, we highlighted the broad range of MAF values observed in all positive EGFR samples.

\subsection{Advantages of Testing Somatic Alterations by NGS on CfDNA}

In the context of a complete molecular profile at diagnosis and during disease progression of NSCLC, the NGS assay provided a broader molecular profiling of patient tumors $(N$ $=615 / 659$; initial diagnosis, $N=249$; progression, $N=366$ ) and missing information or insufficient quantity/quality of cfDNA $(N=44)$ (Fig. 3).

In our routine patient management, both the OncoBEAM $^{\mathrm{TM}}$ EGFR V2 assay and the NGS assay were used to detect oncogene alterations at diagnosis and during disease progression, as the NGS assay detects additional molecular alterations (e.g., ERBB2 amplifications, skipping of MET exon 14, TP53 alterations, as well as others). Given the additional diagnostic criteria needed to further profile patients with $A L K$ resistance or in smokers, additional plasma samples were only analyzed using the NGS 
assay. Except for very few BEAMing-positive cases, samples were not analyzed using the NGS assay for the initial routine management of these patients $(N=14)$. In other rare cases with very low amounts of cfDNA recovered in samples (GE amounts below 2000), NGS testing was not conducted. Finally, we analyzed 531/659 samples using both BEAMing and NGS assays.

Forty-four percent $(N=111 / 249)$ and 50\% $(N=188 / 366)$ of samples at diagnosis and during progression were wildtype. EGFR mutations were identified in $9 \%(N=23 / 249)$ of the patients tested at initial diagnosis and in $21 \%(N=$ 79/366) in cfDNA samples of cases examined during progression. One distinct advantage of using the NGS assays was the identification of rare alterations not covered by BEAMing assays ( $N=13$ cases in the cohort), such as certain sensitizing $E G F R$ exon 19 deletions and primary resistance insertion/duplication alterations in $E G F R$ exon 20 (p.Asn771_His773dup; p.His773_Val774delinsLeuMet; p.Ser768_Asp770dup; p.His773dup; p.Asp770_Asn771insGlyLeu; p.Ser768Ile, c.2303G > T; p.Val774Leu; p.Val786Met). Alterations in genes other than $E G F R$ were found at diagnosis including TP53 $(15 \%, N=37), K R A S$ $(11 \%, N=29)$ and, $M E T(4 \%, N=11)$. In one case, a KRAS p.G12C/EGFR p.V774L co-mutation was observed. At diagnosis and during progression, we observed co-alterations in TP53 and EGFR ( $N=32 / 73$ samples). KRAS mutations were identified at high frequency in patients with NSCLC at diagnosis (11\%) in this cohort (we essentially did not include non-smoker patients, considered as a bias of selection). KRAS positivity in NSCLC was associated with a lack of an efficient response to TKIs [23]. The detection of
KRAS mutations is important to personalize the treatment and improve patient survival. At progression, $21 \%$ of the plasma samples ( 79 alterations, in some cases, two different alterations in the same sample) presented with EGFR alterations. Other genes coding for tyrosine kinase proteins were also mutated, including ERRB2 (2\%), ERBB4 (2\%), and, FGFR3 (1\%). The detection of resistance mechanisms other than EGFR p.T790M and EGFR p.C797S could only be achieved using the NGS assay, to provide a more complete molecular profiling of resistance mechanisms, alongside the OncoBEAM ${ }^{\mathrm{TM}} E G F R$ V2 assay, that clinicians normally encounter during treatment management of patients with NSCLC.

\section{Discussion}

EGFR assays on liquid biopsy routinely enable physicians in the clinic to detect sensitive and resistant genetic alterations at an early stage that may occur during TKI therapy, and to monitor disease progression as evidenced by increasing levels of ctDNA. This can be achieved by frequent plasma sampling and testing of patients at defined intervals of treatment and recovery, alongside imaging, to incorporate analyses of evolving molecular landscapes during treatment. In this setting, the molecular exploration of ctDNA is highly encouraging, especially in advanced NSCLC, where sequential tissue biopsies and tumor tissue testing are impractical, expensive, and may cause undue harm. This is supported by the results of ADAURA (NCT02511106), a phase III, double-blind randomized study assessing the efficacy and safety
Fig. 2 Comparison of positive clinical samples detected with the OncoBEAM ${ }^{\mathrm{TM}}$ EGFR V2 assay and using next-generation sequencing (NGS). Points connected by lines are EGFR alterations found by both BEAMing and NGS assays. $E G F R$ epidermal growth factor receptor

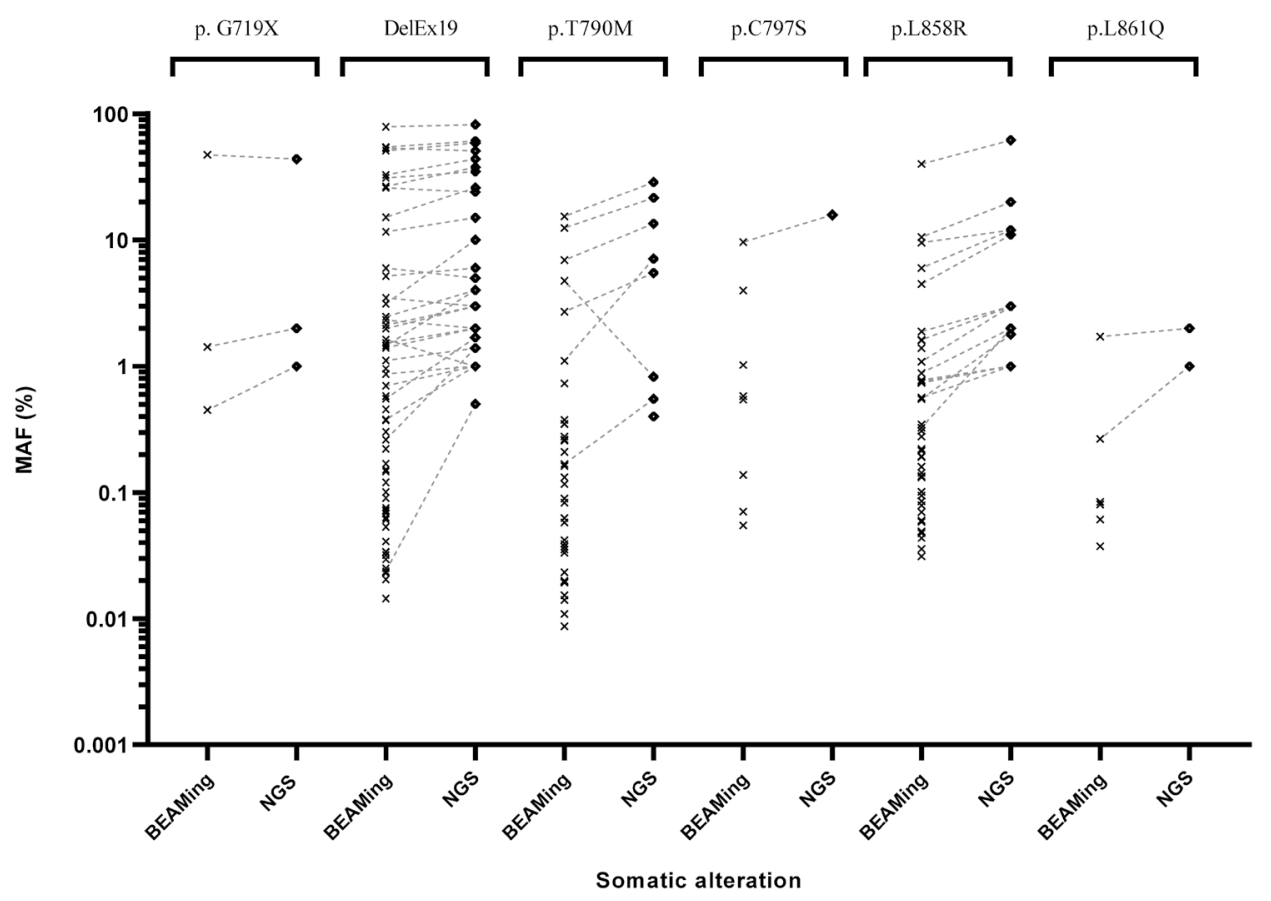


Fig. 3 a Pie charts of somatic alterations found in clinical samples using the nextgeneration sequencing (NGS) assay at diagnosis (DI) and $\mathbf{b}$ during progression $(\mathrm{P})$. In total, clinical samples were analyzed using the NGS assay at diagnosis and during progression. $M A F$ mutant allelic fraction

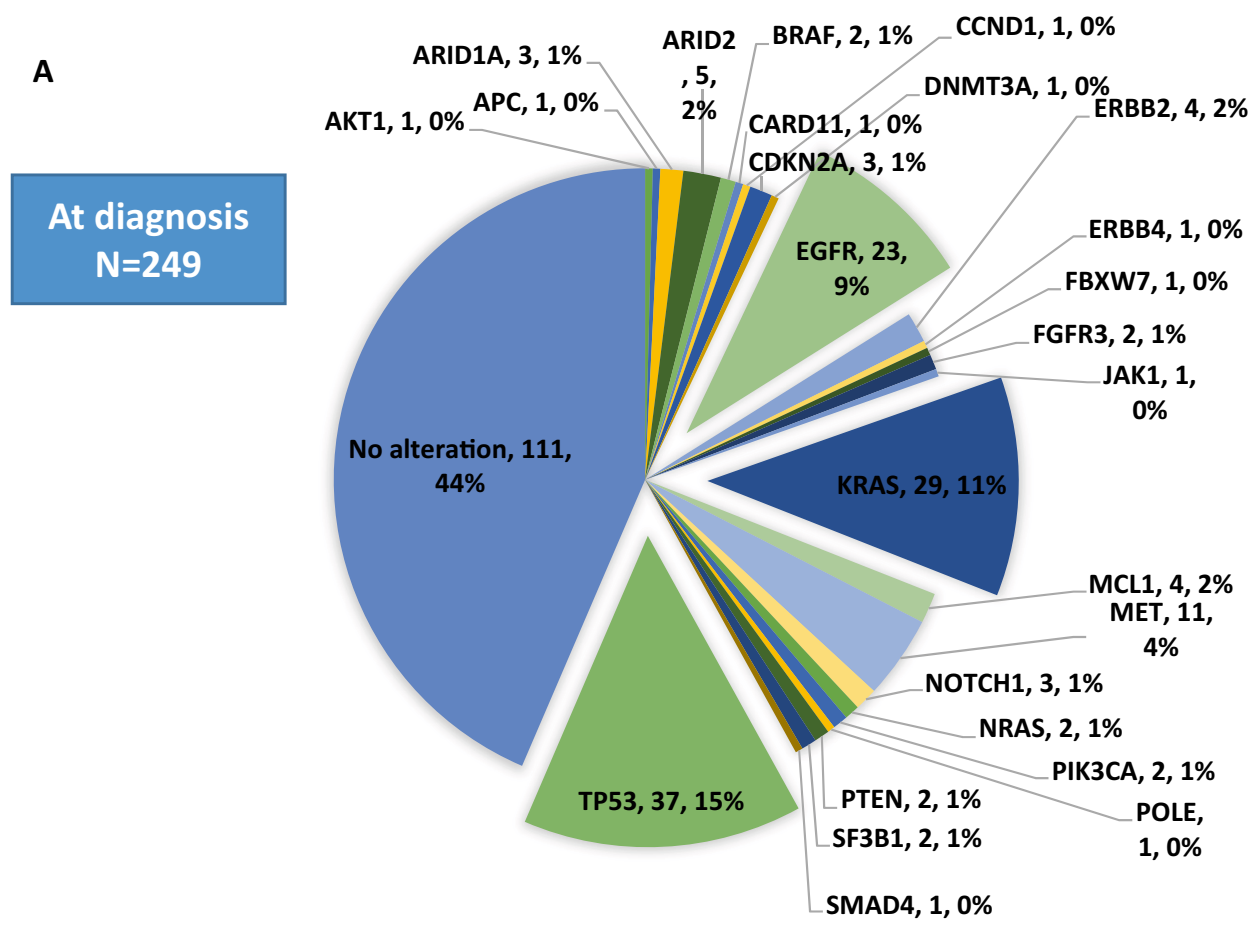

B

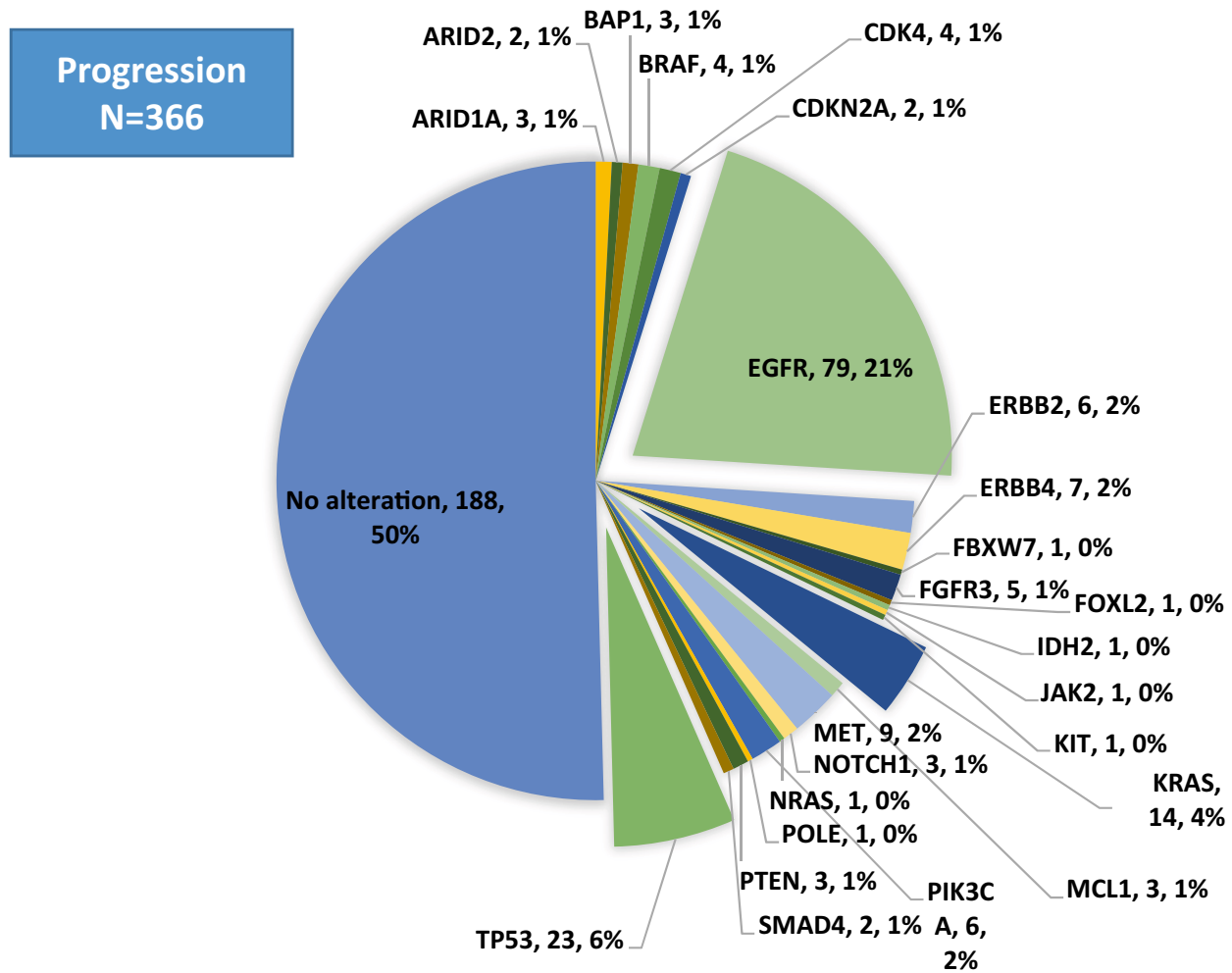

of osimertinib vs placebo in stage IB-IIIA EGFR-mutated NSCLC after complete tumor resection and adjuvant chemotherapy. Adjuvant osimertinib led to a statistically significant and clinically meaningful improvement in progression-free survival. Accordingly, there is now an urgent need to identify all patients with EGFR mutant NSCLC at diagnosis using ultra-sensitive molecular screening tools without sacrificing specificity, especially for patients with unsuccessful tissue biopsies. The diagnosis and characterization of NSCLC is an arduous multi-step process, required in particular for the 
search of oncogenic alterations [24], and while the turnover time has a range of 3-4 weeks with tissue, this is clearly reduced to 1-2 weeks with a cfDNA molecular diagnosis. Tissue biopsy is necessary to identify histological subtypes that influence clinical management and treatment outcome [25] or for staging disease, information that is lacking with liquid biopsy analyses. Therefore, although tissue biopsy remains the gold standard for cancer diagnosis and patient management, the rapid selection required for administering targeted therapy can better be achieved by liquid biopsy testing. The results from such tests, if a well-validated assay with suitable analytical sensitivity and accuracy is used, should streamline the therapy selection process and improve clinical management. Overall, cfDNA testing is a compelling complement to tumor tissue biopsy testing and presents a viable alternative, especially when the quantity/quality of tissue is not sufficient for proper molecular analyses. This is especially true for $E G F R$ TKI first-line therapy, where management decisions for patients with NSCLC and treatment can be initiated within 10 days.

Recent studies have demonstrated a favorable correlation between tumor and paired cfDNA molecular profiles, but the concordance of results varies between 31 and 97\% (depending on pre-analytical and analytical settings) [26]. Metaanalyses have also evaluated the diagnostic value of cfDNA in comparison with tissue mutational testing among 39 studies that encompassed more than 4000 patients. Results showed a sensitivity of $65 \%$ and a specificity of $95 \%$ [27]. Previously, we demonstrated an extremely high degree of concordance between tissue and cfDNA of $98.7 \%$ for the EGFR p.T790M mutation in patients with NSCLC using an OncoBEAM ${ }^{\mathrm{TM}} E G F R$ V1 [11] and of 53\% in a cohort of patients with colon cancer, using the OncoBEAM ${ }^{\mathrm{TM}} R A S$ [28]. Interestingly, lower MAFs of $R A S$ alterations were detected using the BEAMing assay on cfDNA than within tissue biopsies by NGS. Identifying EGFR-positive patients is important, as the response rate to targeted genomic therapy is higher than response rates to first-line chemotherapy or immune checkpoint therapy [29].

In the present study, we validated the clinical performance of the OncoBEAM ${ }^{\mathrm{TM}} E G F R$ V2 assay, a digital PCR test that detects an expanded set of 36 EGFR alterations at very low MAF $(0.02 \%)$. This study is the first to our knowledge that has examined the performance of a large cohort of patients with advanced NSCLC using the OncoBEAM ${ }^{\mathrm{TM}}$ $E G F R$ V2 assay at diagnosis. The results of our study demonstrate a very low background using negative quality control, inferior to ten false-positive mutated beads. All EGFR alterations were detected in positive quality-control samples with a MAF range of $0.1-12 \%$ with accuracy and precision. Moreover, the $0.1 \%$ sensitivity was easily achieved with quality control with only a mean of $3171 \mathrm{GE}$ (corresponding to a $9.5 \mathrm{ng} /$ reaction). A vast majority of clinical samples (more than 65\% at initial diagnosis and during progression) contained sufficient cfDNA (greater than $3000 \mathrm{GE}$ ), to analyze the molecular profile with a clinical sensitivity of at least $0.1 \%$ MAF. Next, extensive testing of patients with NSCLC was performed using the OncoBEAM ${ }^{\mathrm{TM}} E G F R$ V2 assay on cfDNA samples. This analysis demonstrated that EGFR alterations were detected at a prevalence of $11.2 \%$ at diagnosis, which substantiated levels obtained by testing tissue samples for these EGFR alterations, with the majority of results showing the EGFR alterations delEX19 and p.L858R.

During progression, the most common and well-defined resistance mechanism after first-line treatment of patients with NSCLC with gefitinib or erlotinib was the EGFR p.T790M resistance alteration (found at $40.3 \%$, associated with sensitizing EGFR mutations) [30]. In a previously published study, we determined that the EGFR p.T790M mutation was routinely detected in NSCLC plasma samples with a lower limit of detection (analytic sensitivity) of $0.02 \%$ MAF [11]. A study by Chen et al. compared five PCR-based molecular assays using a rather large amount of cfDNA input ( $>25 \mathrm{ng}$ ) for the detection of p.T790M at progression. They reported for a p.T790M mutation a relative clinical sensitivity between $17.5 \%$ and $42.5 \%$ in patients with tissue-confirmed EGFR p.T790M status [31]. The sensitivity of p.T790M detection largely depends on the assay technology used [32] and on cfDNA input. In the present study, we showed that the OncoBEAM ${ }^{\mathrm{TM}} E G F R$ $\mathrm{V} 2$ assay provided reproducible detection of mutations at a very low allelic fraction $(<0.10 \%)$ and with cfDNA levels greater than $3000 \mathrm{GE}$, with great specificity. The positivity threshold to establish the clinically useful cut-off took into account both the mutated clustering shape and the absolute count of mutated beads. In our study, 55\% of patients with $E G F R$ NSCLC cfDNA samples had EGFR alterations below $1 \%$. This finding highlighted the importance of using highly sensitive assays, such as BEAMing, at both the diagnosis of NSCLC for therapy selection and during disease progression to effectively manage patients. In addition to the high specificity/sensitivity of the BEAMing assay, another advantage of using the BEAMing assay in the clinical setting is the assay's rapid turn-around time (2 days for the OncoBEAM EGFR assay vs 5 days for the NGS assay). Therefore, using the BEAMing assay in routine clinical practice can quickly determine whether patients with NSCLC have clinically actionable $E G F R$ gene alterations; this enables rapid decision making in determining the eligibility of patients with NSCLC to receive TKI therapy treatment.

These results support the complementary information given by the NGS assay. Indeed, additional putative resistance mechanisms were found, such as $M E T$ alterations or activation of bypass signaling pathways, including ERBB2 and FGRF3 [10]. Previously, we demonstrated a lower 
sensitivity to detect $R A S$ molecular alterations with NGS compared with BEAMing in cfDNA [28], and as expected, similar conclusions were made with EGFR molecular targets. However, the NGS presents advantages such as a larger coverage, by using a larger gene panel enabling the detection of rare alterations in $E G F R$ or in other genes. Our home-design gene panel based on capture technology was developed to evaluate gene amplifications (in addition to mutation, insertion, and deletion detection), which are frequent resistance mechanisms. At initial diagnosis, KRAS mutations were found in $25 \%$ of cases [33], which is higher than in the present study (11\%), though patients with known KRAS mutations at initial diagnosis in tissue biopsies were excluded from our current cohort. During progression, few secondary resistance mechanisms were found after TKI treatment that involved alternative tyrosine kinase signaling pathways, including ERRB2, ERBB4, and FGFR.

\section{Conclusions}

Our continued commitment to using an effective combination of focal OncoBEAM ${ }^{\mathrm{TM}} E G F R$ V2 testing, as well as an expanded NGS panel, provides insights into the diversity of resistance mechanisms. Rapid identification of such genomic resistance mechanisms is still needed in routine clinical practice.

Supplementary Information The online version contains supplementary material available at https://doi.org/10.1007/s40291-021-00515-9.

Acknowledgements The authors thank Astra-Zeneca and Sysmex Inostics for their continued financial support of this clinical diagnostic study.

Author contributions JG, SC, and LP conceived the study. GL, FG, and JB performed experimental assays. JG, DB, AG, GL, FG, JB, and LP analyzed the results. All authors reviewed the final version of the manuscript.

\section{Declarations}

Funding This work was supported by research grants from AstraZeneca (London, UK).

Conflict of interest Frank Holtrup, Frederick S. Jones, and Daniel L. Edelstein are employees of Symex Inostics. Sébastien Couraud has no potential conflict of interest with Astra Zeneca, Sysmex Inostics, Roche, and Boehringer Ingelheim that is directly relevant to the content of this article. Jessica Garcia, Arnaud Gauthier, Gaëlle Lescuyer, David Barthelemy, Florence Geiguer, Julie Balandier, Mickael Duruisseau, Emmanuel Grolleau, Claire Rodriguez-Lafrasse, Patrick Merle, Sébastien Couraud, and Léa Payen have no conflicts of interest that are directly relevant to the content of this article.

Ethics approval All samples sent to the laboratory for EGFR routine screening within cfDNA between September 2018 and February 2020 were included in this retrospective cohort study. For each sample, medical data were collected through mandatory prescription sheet attached to each sample and edited by the prescribing physician.

Consent to participate All participants consented to participate in the study.

Consent for publication Not applicable.

Code availability Not applicable.

Availability of data and material The datasets used or analyzed during the current study are available from the corresponding author on reasonable request.

Open Access This article is licensed under a Creative Commons Attribution-NonCommercial 4.0 International License, which permits any non-commercial use, sharing, adaptation, distribution and reproduction in any medium or format, as long as you give appropriate credit to the original author(s) and the source, provide a link to the Creative Commons licence, and indicate if changes were made. The images or other third party material in this article are included in the article's Creative Commons licence, unless indicated otherwise in a credit line to the material. If material is not included in the article's Creative Commons licence and your intended use is not permitted by statutory regulation or exceeds the permitted use, you will need to obtain permission directly from the copyright holder. To view a copy of this licence, visit http://creativecommons.org/licenses/by-nc/4.0/.

\section{References}

1. Wood SL, Pernemalm M, Crosbie PA, Whetton AD. Molecular histology of lung cancer: from targets to treatments. Cancer Treat Rev. 2015;41(4):361-75. https://doi.org/10.1016/j. ctrv.2015.02.008

2. Paez JG, Jänne PA, Lee JC, Tracy S, Greulich H, Gabriel S, et al. EGFR mutations in lung cancer: correlation with clinical response to gefitinib therapy. Science. 2004;304(5676):1497. https://doi. org/10.1126/science.1099314.

3. Rosell R, Carcereny E, Gervais R, Vergnenegre A, Massuti B, Felip E, et al. Erlotinib versus standard chemotherapy as firstline treatment for European patients with advanced EGFR mutation-positive non-small-cell lung cancer (EURTAC): a multicentre, open-label, randomised phase 3 trial. Lancet Oncol. 2012;13(3):239-46. https://doi.org/10.1016/S1470 $-2045(11) 70393-X$

4. Solomon BJ, Mok T, Kim D-W, Wu Y-L, Nakagawa K, Mekhail $\mathrm{T}$, et al. First-line crizotinib versus chemotherapy in ALK-positive lung cancer. N Engl J Med. 2014;371(23):2167-77. https://doi. org/10.1056/NEJMoa1408440.

5. Planchard D, Besse B, Groen HJM, Souquet P-J, Quoix E, Baik $\mathrm{CS}$, et al. Dabrafenib plus trametinib in patients with previously treated BRAF(V600E)-mutant metastatic non-small cell lung cancer: an open-label, multicentre phase 2 trial. Lancet Oncol. 17(7):984-93. https://doi.org/10.1016/s1470-2045(16)30146-2.

6. Mok TS, Wu Y-L, Ahn M-J, Garassino MC, Kim HR, Ramalingam SS, et al. Osimertinib or platinum-pemetrexed in EGFR T790M-positive lung cancer. N Engl J Med. 2017;376(7):629-40. https://doi.org/10.1056/NEJMoa1612674.

7. Li K, Yang M, Liang N, Li S. Determining EGFR-TKI sensitivity of G719X and other uncommon EGFR mutations in non-small cell lung cancer: perplexity and solution (review). Oncol Rep. 2017;37(3):1347-58. https://doi.org/10.3892/or.2017.5409. 
8. D’Angelo SP, Pietanza MC, Johnson ML, Riely GJ, Miller VA, Sima CS, et al. Incidence of EGFR exon 19 deletions and L858R in tumor specimens from men and cigarette smokers with lung adenocarcinomas. J Clin Oncol. 2011;29(15):2066-70. https://doi. org/10.1200/jco.2010.32.6181.

9. Carey KD, Garton AJ, Romero MS, Kahler J, Thomson S, Ross $\mathrm{S}$, et al. Kinetic analysis of epidermal growth factor receptor somatic mutant proteins shows increased sensitivity to the epidermal growth factor receptor tyrosine kinase inhibitor, erlotinib. Cancer Res. 2006;66(16):8163-71. https://doi.org/10.1158/00085472.can-06-0453.

10. Rotow J, Bivona TG. Understanding and targeting resistance mechanisms in NSCLC. Nat Rev Cancer. 2017;17(11):637-58. https://doi.org/10.1038/nrc.2017.84.

11. Garcia J, Wozny AS, Geiguer F, Delherme A, Barthelemy D, Merle P, et al. Profiling of circulating tumor DNA in plasma of non-small cell lung cancer patients, monitoring of epidermal growth factor receptor p.T790M mutated allelic fraction using beads, emulsion, amplification, and magnetics companion assay and evaluation in future application in mimicking circulating tumor cells. Cancer Med. 2019;8(8):3685-97. https://doi. org/10.1002/cam4.2244.

12. Rosell R, Karachaliou N. Implications of blood-based T790M genotyping and beyond in epidermal growth factor receptor-mutant non-small-cell lung cancer. J Clin Oncol. 2016;34(28):3361-2. https://doi.org/10.1200/jco.2016.68.3458.

13. Planchard D, Popat S, Kerr K, Novello S, Smit EF, Faivre-Finn C, et al. Metastatic non-small cell lung cancer: ESMO clinical practice guidelines for diagnosis, treatment and follow-up. Ann Oncol. 2018;29:iv192-237. https://doi.org/10.1093/annonc/mdy275.

14. Novello S, Barlesi F, Califano R, Cufer T, Ekman S, Levra MG, et al. Metastatic non-small-cell lung cancer: ESMO clinical practice guidelines for diagnosis, treatment and follow-up. Ann Oncol. 2016;27:v1-27. https://doi.org/10.1093/annonc/mdw326.

15. Sun W, Yuan X, Tian Y, Wu H, Xu H, Hu G, et al. Non-invasive approaches to monitor EGFR-TKI treatment in non-small-cell lung cancer. J Hematol Oncol. 2015;8:95. https://doi.org/10.1186/ s13045-015-0193-6.

16. Mader S, Pantel K. Liquid biopsy: current status and future perspectives. Oncol Res Treat. 2017;40(7-8):404-8. https://doi. org/10.1159/000478018.

17. Wang J, Chang S, Li G, Sun Y. Application of liquid biopsy in precision medicine: opportunities and challenges. Front Med. 2017;11(4):522-7. https://doi.org/10.1007/s11684-017-0526-7.

18. Bronkhorst AJ, Ungerer V, Holdenrieder $\mathrm{S}$. The emerging role of cell-free DNA as a molecular marker for cancer management. Biomol Detect Quantif. 2019;17:100087. https://doi.org/10.1016/j. bdq.2019.100087.

19. Douillard JY, Ostoros G, Cobo M, Ciuleanu T, McCormack R, Webster A, et al. First-line gefitinib in Caucasian EGFR mutation-positive NSCLC patients: a phase-IV, open-label, single-arm study. Br J Cancer. 2014;110(1):55-62. https://doi.org/10.1038/ bjc. 2013.721.

20. Lim C, Tsao MS, Le LW, Shepherd FA, Feld R, Burkes RL, et al. Biomarker testing and time to treatment decision in patients with advanced nonsmall-cell lung cancer. Ann Oncol. 2015;26(7):1415-21. https://doi.org/10.1093/annonc/mdv208.
21. Chouaid C, Dujon C, Do P, Monnet I, Madroszyk A, Le Caer H, et al. Feasibility and clinical impact of re-biopsy in advanced non small-cell lung cancer: a prospective multicenter study in a realworld setting (GFPC study 12-01). Lung Cancer. 2014;86(2):1703. https://doi.org/10.1016/j.lungcan.2014.08.016.

22. Oxnard GR, Thress KS, Alden RS, Lawrance R, Paweletz CP, Cantarini M, et al. Association between plasma genotyping and outcomes of treatment with osimertinib (AZD9291) in advanced non-small-cell lung cancer. J Clin Oncol. 2016;34(28):337-82. https://doi.org/10.1200/JCO.2016.66.7162.

23. Del Re M, Crucitta S, Gianfilippo G, Passaro A, Petrini I, Restante $\mathrm{G}$, et al. Understanding the mechanisms of resistance in EGFRpositive NSCLC: from tissue to liquid biopsy to guide treatment strategy. Int J Mol Sci. 2019;20(16):3951. https://doi.org/10.3390/ ijms20163951.

24. Bubendorf L, Lantuejoul S, de Langen AJ, Thunnissen E. Nonsmall cell lung carcinoma: diagnostic difficulties in small biopsies and cytological specimens. Eur Respir Rev. 2017;26(144):170007. https://doi.org/10.1183/16000617.0007-2017.

25. McLean AEB, Barnes DJ, Troy LK. Diagnosing lung cancer: the complexities of obtaining a tissue diagnosis in the era of minimally invasive and personalised medicine. J Clin Med. 2018;7(7):163. https://doi.org/10.3390/jcm7070163.

26. Wu Y-L, Sequist LV, Hu C-P, Feng J, Lu S, Huang Y, et al. EGFR mutation detection in circulating cell-free DNA of lung adenocarcinoma patients: analysis of LUX-Lung 3 and 6 . Br J Cancer. 2017;116(2):175-85. https://doi.org/10.1038/bjc.2016.420.

27. Mlika M, Dziri C, Zorgati MM, Ben Khelil M, Mezni F. Liquid biopsy as surrogate to tissue in lung cancer for molecular profiling: a meta-analysis. Curr Respir Med Rev. 2018;14(1):48-60. https://doi.org/10.2174/1573398X14666180430144452.

28. Garcia J, Forestier J, Dusserre E, Wozny AS, Geiguer F, Merle P, et al. Cross-platform comparison for the detection of RAS mutations in cfDNA (ddPCR Biorad detection assay, BEAMing assay, and NGS strategy). Oncotarget. 2018;9(30):21122-31. https://doi. org/10.18632/oncotarget.24950.

29. Liang W, Zhang Y, Kang S, Pan H, Shao W, Deng Q, et al. Impact of EGFR mutation status on tumor response and progression free survival after first-line chemotherapy in patients with advanced non-small-cell lung cancer: a meta-analysis. J Thorac Dis. 2014;6(9):1239-50. https://doi.org/10.3978/j. issn.2072-1439.2014.07.33.

30. Yu HA, Arcila ME, Rekhtman N, Sima CS, Zakowski MF, Pao W, et al. Analysis of tumor specimens at the time of acquired resistance to EGFR-TKI therapy in 155 patients with EGFR-mutant lung cancers. Clin Cancer Res. 2013;19(8):2240-7. https://doi. org/10.1158/1078-0432.ccr-12-2246.

31. Chen Y-L, Lin C-C, Yang S-C, Chen W-L, Chen J-R, Hou Y-H, et al. Five technologies for detecting the EGFR T790M mutation in the circulating cell-free DNA of patients with non-small cell lung cancer: a comparison. Front Oncol. 2019;9:631. https://doi. org/10.3389/fonc.2019.00631.

32. Diaz LA Jr, Bardelli A. Liquid biopsies: genotyping circulating tumor DNA. J Clin Oncol. 2014;32(6):579-86. https://doi. org/10.1200/jco.2012.45.2011.

33. Kempf E, Rousseau B, Besse B, Paz-Ares L. KRAS oncogene in lung cancer: focus on molecularly driven clinical trials. Eur Respir Rev. 2016;25(139):71-6. https://doi.org/10.1183/16000 617.0071-2015. 


\section{Authors and Affiliations}

\section{Jessica Garcia ${ }^{1,2}$ - Arnaud Gauthier ${ }^{1}$. Gaëlle Lescuyer ${ }^{1,2}$ - David Barthelemy ${ }^{1,2}$. Florence Geiguer ${ }^{1,2}$. Julie Balandier ${ }^{1,2}$. Daniel L. Edelstein ${ }^{3}$. Frederick S. Jones ${ }^{3} \cdot$ Frank Holtrup $^{3} \cdot$ Mickael Duruisseau $^{4}$. Emmanuel Grolleau $^{6}$. Claire Rodriguez-Lafrasse ${ }^{1} \cdot$ Patrick Merle $^{5} \cdot$ Sébastien Couraud $^{2,6,7} \cdot$ Léa Payen $^{1,2,8} \mathbb{B}$}

1 Laboratoire de Biochimie et Biologie Moléculaire, Groupe Hospitalier Sud, Hospices Civils de Lyon, Pierre-Bénite, France

2 Hospices Civils de Lyon Cancer Institute, CIRculating CANcer (CIRCAN) Program, Pierre-Bénite, France

3 Life Sciences Medical Affairs and Research and Development Sysmex Inostics, GmBH, Hamburg, Germany

4 Service de Pneumologie, Groupement Hospitalier Est, Hospices Civils de Lyon, Bron, France
Service de Pneumologie, Centre Hospitalier Gabriel Montpied, Clermont-Ferrand, France

6 Service de Pneumologie Aigue Spécialisée et Cancérologie Thoracique, Groupement Hospitalier Sud, Institut de Cancérologie des Hospices Civils de Lyon, Pierre-Bénite, France

7 EMR 3738 Ciblage Thérapeutique en Oncologie, Faculté de Médecine Lyon-Sud, Université de Lyon, Oullins, France

8 Hospices Civils de Lyon, Centre Hospitalier Lyon Sud, 165, Chemin du Grand Revoyet, 69495 Pierre-Bénite, France 